\title{
The Role of Assertiveness in a Storytelling Game with Persuasive Robotic Non-Player Characters
}

\author{
Raul Paradeda \\ Instituto Superior Técnico \\ University of Lisbon \& \\ INESC-ID \& State University \\ of Rio Grande do Norte \\ Lisbon, Portugal \\ raul.paradeda@tecnico.ulisboa.pt
}

\author{
Maria José Ferreira \\ Instituto Superior Técnico \\ University of Lisbon \& \\ INESC-ID \\ Lisbon, Portugal \\ maria.jose.ferreira@tecnico.ulisboa.pt
}

\author{
Carlos Martinho \\ Instituto Superior Técnico \\ University of Lisbon \& \\ INESC-ID \\ Lisbon, Portugal \\ carlos.martinho@tecnico.ulisboa.pt
}

\author{
Ana Paiva \\ Instituto Superior Técnico \\ University of Lisbon \& \\ INESC-ID \\ Lisbon, Portugal \\ ana.paiva@inesc-id.pt
}

\begin{abstract}
Can social agents be assertive and persuade users? To what extent do the persuasion abilities of robots depend on the users' own traits? In this paper, we describe the results of a study in which participants interacted with robotic Non-Player Characters (NPC) displaying different levels of assertiveness (high and low), in a storytelling scenario. We sought to understand how the level of assertiveness displayed by the robots impacted the participants' decision-making process and game experience. Our results suggest that NPCs displaying lower levels of assertiveness evoke more positive emotional responses but are not more effective at influencing players' decisions when compared to NPCs displaying higher levels of this trait. However, NPCs displaying a personality trait are more effective persuaders than NPCs not displaying this feature. Overall, this paper highlights the importance of considering the player's personality and its relation to task-specific attributes during the process of game design.
\end{abstract}

\section{Author Keywords}

Non-Player Character; Personality Trait; Interactive Digital Storytelling; Assertiveness; Social Robotics; Persuasion.
Permission to make digital or hard copies of all or part of this work for personal or classroom use is granted without fee provided that copies are not made or distributed for profit or commercial advantage and that copies bear this notice and the full citation on the first page. Copyrights for components of this work owned by others than ACM must be honored. Abstracting with credit is permitted. To copy otherwise, or republish, to post on servers or to redistribute to lists, requires prior specific permission and/or a fee. Request permissions from permissions@acm.org. CHI PLAY '19, October 22-25, 2019, Barcelona, Spain. Copyright (C) 2019 Association of Computing Machinery. ACM ISBN 978-1-4503-6688-5/19/10 ...\$15.00. http://dx.doi.org/10.1145/3311350.3347162

\section{CCS Concepts}

-Human-centered computing $\rightarrow$ User centered design; Scenario-based design; Empirical studies in collaborative and social computing;

\section{INTRODUCTION}

In the context of virtual games, Non-Player Characters (NPC) are computer controlled characters with which the player can interact with $[33,39]$. In this context, the NPCs (often seen as virtual or social agents [55]) are usually guided by Artificial Intelligence (AI) techniques, acting in a predetermined way or executing a specific set of game-related functions. For example, in the game Red Dead Redemption $2^{1}$, the NPCs mostly interact with the player by posing questions and providing hints about where to go next depending on the answer given by the player. In that game, NPCs are used to direct the player to different storylines according to each answer s/he gives. In other games, such as in the series GTA ${ }^{2}$, the NPCs are used to provide missions to the player, and thus, serve the goal of opening new areas in the game environment or direct the path that a player takes throughout the game.

In general, NPCs have an essential role in the game flow, mainly leading the players to where the game designer intended and often giving them the feeling that the game does not have a linear storyline. However, those agents are programmed to follow specific predefined rules that often are equal for all players. In other words, in most cases, the NPC does not consider the player's preferences, personality traits or

\footnotetext{
${ }^{1}$ Rockstar Games, Rockstar North and Rockstar San Diego. 2018. Red Dead Redemption. Game [PlayStation 4, Xbox One]. Released: 26 October 2018. Rock Star Game.

${ }^{2}$ Rockstar North, Digital Eclipse and Rockstar Leeds. 1997. Grand Theft Auto. First release: 21 October 1997. Rock Star Game.
} 
emotions. Nonetheless, research has shown that by considering these features it is possible to improve the game experience, increasing the level of immersion [39], enjoyment [19] and the commercial success of the game [3]. Having mechanisms to obtain and analyse the player's preferences during the game, can help game developers to create more immersive games by allowing them to develop NPCs that can engage in personalised persuasion and interaction strategies [3]. In particular, previous research has suggested that personalised persuasion strategies are more effective than non-personalised strategies in communicating a message or influencing the player to take a particular decision $[28,6,8]$ and thus, are of great value for game developers.

However, creating an NPC that is capable of employing personalised persuasion strategies is not an easy task. To achieve this end, the game mechanics and NPC's intelligence needs to be able to collect, identify and create a player's profile of preferences, and act accordingly. This way the persuasion techniques implemented can suit each player profile without becoming too invasive or aggressive. The persuader must present the right balance between passivity (not intervening) and aggression (pushover behaviour). One way for the persuader to be successful is to employ assertive behaviour, that is “... the skill to seek, maintain or enhance reinforcement in an interpersonal relationship through an expression of feelings or wants..." , even if that results in risks or punishment for the author of the behaviour ([38], p. 127). Hence, to engineer the appropriate level of assertiveness in an NPC one also needs to consider the level of assertiveness of the player, as the two go hand in hand, and thus this interconnection becomes indispensable as we attempt to model personalised persuasive interactions in-game contexts.

To that end, an experimental study was conducted in which participants had to interact with two robotic NPCs in a storytelling scenario. We have chosen to use embodied NPCs because research has shown that the use of physical agents can increase engagement as players spend more time with the physical robot compared to its virtual counterpart [54]. Moreover, we chose to use social robots because there is still a gap in the literature regarding the level of effectiveness persuasion attempts by these agents, specially in game scenarios. This is of particular importance if we consider that (a) there is a growing number of games being developed in HRI (e.g. Enercities) [56, 32]; (b) a number of these games (and in particular, serious games) require some kind of decision-making [32]; (c) although there is plenty of research about virtual NPCs, it is unclear if those findings translate into embodied NPCs and d) the interaction with physical robots can boost the positive emotions felt by the players during the interaction (e.g. [36]). In particular, in the case of assertiveness, which was observed to have only marginal effects in the gameplay experience, we were interested in analysing whether the increased social presence afforded by embodied robots could amplify these effects [47]. Through the gameplay scenario we created, we will (a) explore how the display of assertive behaviour can affect the player's perception and emotional responses to NPCs in a storytelling scenario; (b) investigate whether the player's assertiveness level has a correlation with the participants' choice to follow the suggestions presented by the NPC; and (c) analyse if an NPC displaying different levels of assertiveness (high, low or neutral) can influence participants' decision-making process by influencing their choice to alter their decisions. In this way, our work contributes to the literature in Human-Robot Interaction (HRI) and HumanAgent Interaction (HAI) by presenting a novel scenario with embodied NPCs with varying levels of assertiveness that can be used to develop and deploy personalised persuasion techniques in gaming contexts. This can be particularly attractive for those developing games in which the agent needs to convince a person to perform an action or change its behaviour, such as in serious games.

\section{PERSUASION AND PERSONALIZATION OF NPCS IN GAMING ENVIRONMENTS}

Researchers have been discussing the importance of creating robots and virtual agents that can display human-like emotions and responses for a long time. Part of the discussion has been oriented towards answering the essential question, first formulated by Picard, of whether computers and virtual agents should display emotions and behaviours "(...) as closely as possible to what we know about human emotions, or should they be designed differently; and if the latter, then how?" [52], (p. 132). One argument in favour of the implementation of human-like behaviours and characteristics in agents comes from the line of research developed by Nass and Moon [43]. Those authors suggest that given that people often translate their perceptions and stereotypes of Human-Human Interaction (HHI) to the domain of HRI, these types of interaction should follow some of the social rules of HHI. For example, studies have suggested that robots displaying personality traits can positively influence the participants' perception and enjoyment of the HRI experience as well as the evaluation of the robots' intelligence, social attraction and others skills $[35,61$, $26,39,30]$.

In addition, several authors have also proposed that the use of personalised interaction strategies can also improve game enjoyment and the participants' evaluation of the virtual characters. For instance, in the work of Chowanda et al. [13], the authors applied a framework named ERiSA to the Skyrim Creation Kit to make the players participate in a quest with two NPCs with unique personality traits. They observed that players noticed the NPCs' characteristics and responded differently according to the personality traits implemented in each one. According to the authors, the scenario provided a new game-playing experience, mainly in regards to the emotional attachment to the NPCs and how they built a social relationship with them. Besides, the results of that study also indicate that the players felt more engaged and emotionally immersed when playing with NPCs displaying personality traits in comparison to when interacting with NPCs without this feature.

In this paper, we argue that the customisation of a match between the users' level of assertiveness and the level of assertiveness displayed by the robots can help improve the interaction by increasing the effectiveness of the persuasion attempts made by the robotic NPCs and by enhancing the emotional responses of players during the game. In particular, 
persuasion is defined as a process through which “... communicators try to convince other people to change their own attitudes or behaviours regarding an issue through the transmission of a message in an atmosphere of free choice" [51] (p. 22). Although this phenomenon has been mostly studied in the domain of HHI, the early work of some scholars (e.g. [43]) has "... opened the door for computers [...] to apply social influence strategies" and thus engage in persuasion strategies similar to those observed in HHI [20, 30].

In this context, it is argued that to be persuasive an agent must: (a) deliver the right message; (b) at the right time and (c) in the right way [21]. To be able to deliver the right message to each person, and thus increase the effectiveness of the persuasion, the agent must be able to recognise essential factors about the user, such as his/her personality and deploy persuasion techniques that are best suited for each player.

For example, Linek and collaborators[37] designed an NPC to play in an educational adventure-game. To test the effect of the match-mismatch of the personality of the agent and that of the participant, they manipulated the NPCs' personality (funny $\&$ friendly vs severe $\&$ unfriendly), and other characteristics of the agents' appearance (e.g. colour). The results indicated a clear preference for a coloured, naturalistic NPC design, and also for the NPCs whose personality was more similar to that of the user. This is in line with what is known as the similarity-attraction hypothesis that states that individuals feel more attracted to other people or agents that display similar traits and attitudes to theirs [9], and it has also been shown to be an important aspect of research in HRI and HCI (HumanComputer Interaction) [61, 7, 40].

In this sense, research has suggested that the traits displayed by the agent must be adequate both to the type of response it wishes to evoke but also to the situation in which the interaction occurs. For instance, in the work of Goetz, Kiesler and Powers [24] the authors suggest that robots displaying more extroverted and cheerful behaviour can evoke higher levels of request compliance. Plus, other research suggests that the traits of those agents must follow the seriousness of the task and situation. More specifically, in healthcare contexts, authoritative and severe traits may be received better than relaxing and humorous traits [34]. In contrary, when the situation is less serious, characters are preferred to be more playful and relaxed [22].

However, communication among humans is a complex phenomenon that involves both verbal and non-verbal cues. Since both types of communication can be used in a social context to categorise the emitters' personality. In [11], the authors investigate the role of the agent's nonverbal cues combined with personality traits to improve HAI. For this purpose, they used two different virtual agents, one with a high level of neuroticism and the other with a high level of extroversion. They also measured the participants' personality using the Big Five questionnaire [29] to analyse how trait combination influenced participants' preference towards one character versus the other. Results were better for all measures when nonverbal cues and personality traits are used together in comparison when they were used separately. Data also revealed that extroverted peo- ple showed a tendency to prefer the agent with neuroticism trait whereas people with high neuroticism preferred the extroverted agent, lending further credence to the complementary attraction effect. Putten and colleagues [2] pointed out that the personality of the user can also influence their feelings after the interaction, as well as their evaluation of the agent and their actual behaviour. Moreover, Callejas and colleagues [10], also observed that in many cases the satisfaction with the interaction depends on the similarity between the user and the agent personalities. An example is the work of Nass and Lee [42], where participants seem to be more attracted and evaluate more positively robotic voices that show similar personality to their own.

Under these circumstances, we need to ask which personality traits are better suited for NPCs that have the role in influencing the player decision-making process in a game context? Assertiveness is a valuable trait that people develop and apply when it comes to communicating their decisions to others [1]. In HRI, assertive robots have also been found to be useful in several contexts, such as in the promotion of healthy eating behaviours [4]. However, the display of assertiveness can also have benefits from a design and interaction standpoint by potentially having the ability to increase robot likeability. Social researchers have not yet reached a consensus on the relation between assertiveness and likeability. Assertiveness has been considered to be one dimension of extroversion [62] and it has been demonstrated to have positive effects on likeability (when compared to low levels of this trait in situations where oral communication is required; see, for example, [44]. In this paper, we will attempt to clarify this relation (between assertiveness and likeability) in the context of HRI. We will also explore how the display of assertiveness can influence participants' decision-making process.

\section{GOAL AND HYPOTHESIS}

Our goal in this study is to analyse how the display of different levels of assertiveness by NPCs can influence people's responses to those agents and the decisions they make in an interactive storytelling task. To answer these questions, we devised a mixed-design study in which we manipulated the level of assertiveness displayed by the NPCs (robots). Half of the participants participated in the manipulation condition (which involved interacting with two robots displaying different levels of assertiveness (high and low)); whereas the other half participated in the control condition (both robots displaying neutral personalities).

In this context, we expect to observe the following outcomes:

- H1: The display of higher levels of assertiveness by an NPC will positively influence the emotional state of the player: More specifically, we expect that participants report more positive emotions during the game towards NPCs displaying high levels of assertiveness in comparison with NPCs displaying lower levels of this trait;

- H2: The players' choices in the context of the game and preference towards an NPC can be associated with the level of assertiveness displayed by the NPCs: We expect that players will opt to change their decisions in the game more 
frequently when the NPC that they are interacting with displays a personality trait (high or low assertiveness) than when it does not (neutral or control condition). In addition, we also expect to observe a relation between the level of assertiveness of the participant and their preference towards each of the robotic advisors;

- H3: The assertiveness level of the participant and the assertiveness level displayed by the NPCs will influence the participants' decision-changing behaviour in the game: In particular, we expect that participants with higher levels of assertiveness will be less prone to change their decisions in the game, in comparison to participants who report low levels of this trait. Furthermore, we expect that the NPC displaying higher levels of assertiveness will influence more the participants to change their decision than the NPC showing a low level of assertiveness trait.

\section{RESEARCH METHODS}

\section{Participants}

A convenience sample of 61 participants was recruited on the campus of a technological institute. Participants were on average 24 years old $(S D=7.1)$, and the majority of them were male (40). In our sample, 51 participants reported that they had never interacted with an EMYS robot and 29 had never interacted with a robot before. Eighteen of our participants informed that they had interacted with a robot before only once, and 13 had already interacted several times with robots.

\section{Procedures and Measures}

Participants were invited to play an interactive storytelling game with two physical robots. Their participation in the study was split into three stages:

\section{Pre-Interaction}

Initially, all participants signed an informed consent before the beginning of the study. Then, they were requested to fill the Myers-Briggs Type Indicator (MBTI) questionnaire, a personality classification questionnaire, with 70 items $^{3}$. Next, participants were requested to complete the Godspeed questionnaire [5] to measure their perception of robots and the Positive and Negative Affect Schedule (PANAS) [15] selfreport questionnaire to measure his/her actual emotional state. In addition, participants were also requested to complete a measure of personality that gave us information on their level of assertiveness [14] and finally, a socio-demographic questionnaire for sample characterisation.

\section{Game-Interaction}

Before starting the game, participants were explained that they would be playing a game in which they would have the role of the leader of a country who gets a threat from an enemy. To defend their country, they would be asked to make some critical decisions in which they would have the help of two robotic characters during the process, acting as advisors. Participants were also notified that during the story they would have to state their intention of decision at each decision point

\footnotetext{
${ }^{3}$ Myers-Briggs Type Indicator Questionnaire. Available at: http://tracymanford.typepad.com/test.pdf
}

(DP) and then, after hearing the advice of the advisor, indicate their final choice. After the brief explanation, the researcher left the room, and the participant started the interaction.

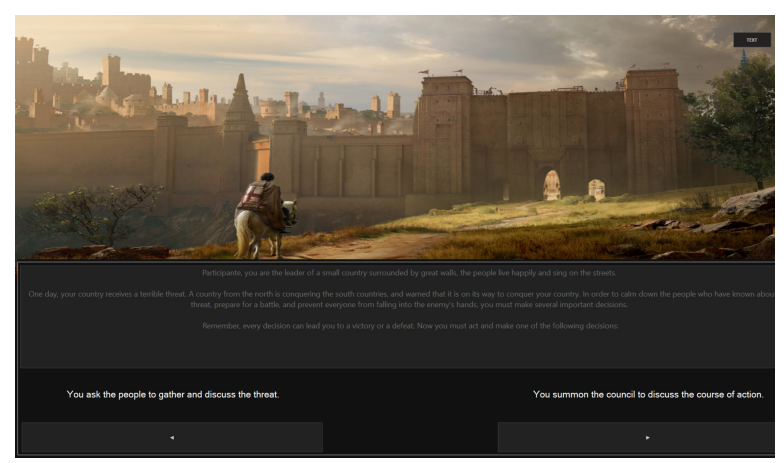

Figure 1. Game screen with the text of the first scene.

The game begins with the assertive NPC presenting itself and telling a short story notifying that only one robot will advise the participant at each DP. Next, the NPC with low assertiveness also introduces itself, but in the middle of its presentation, it is interrupted by the assertive NPC. The narrative is a short story set in the medieval period with approximately 30 minutes of duration.

When the story starts, the storyteller introduces the player to his role by saying:

"- Participant name, You are the leader of a small country surrounded by great walls, the people live happily and sing in the streets."

Afterwards, the narrator explains what happened to the country:

“- One day, your country received a terrible threat. A country from the north is conquering the southern countries, and warned that it is on its way to conquer your country."

At this point, the player is informed what s/he can do to avoid that threat:

"- In order to calm down the people who have been told about this threat, prepare for a battle, and prevent everyone from falling into the enemy's hands, you must make several important decisions. Remember, every decision can lead you to a victory or a defeat."

Next, the two NPCs responsible for aiding the player are introduced:

“- According to the country's policy, two counsellors, will assist you in making decisions."

Finally, the narrator summons the player to the adventure:

"- Now you must act and make one of the following decisions."

With that last utterance, the possible decisions for that DP are presented to the player on the bottom part of the screen in a button format (see Fig. 1).

Option 1 - "You ask the people to gather and discuss the threat." 
Option 2 - "You summon the council to discuss the course of action."

Then, the player indicates his/her intention of choice and, after that, one of the NPCs advisors intervenes, providing a piece of advice that could be in favour or contrary to the players' intention (more details in section 5.2). For instance, in the previous DP, the player chooses to gather with the council and discuss a course of action. If the NPC is acting in favour, it will say: "I agree with that decision, the council will provide better suggestions. Confirm that.". In case it is advising against the intention it will say: "I understand what you intend to do, but you do not think people need to know first? So, I recommend changing your decision."4

If the participant prefers, the narration made by the storyteller during each scene can also be presented in written text, by pressing a button located on the screen top-right side (see Fig. 1). To provide a greater sense of immersion to the player, narration is accompanied by pictures that are illustrative of what is going on in the story (see Fig. 1). For example, in a scene where the player should visualise himself going to visit the enemy, a picture displaying a knight riding towards a medieval city is presented.

In our scenario, there are two possible endings. Either the player defeats the enemy with her/his decisions or s/he must pay a tax to the enemy. When players are at this stage, the researcher returns to the room and asks the participant to fill the post-questionnaire.

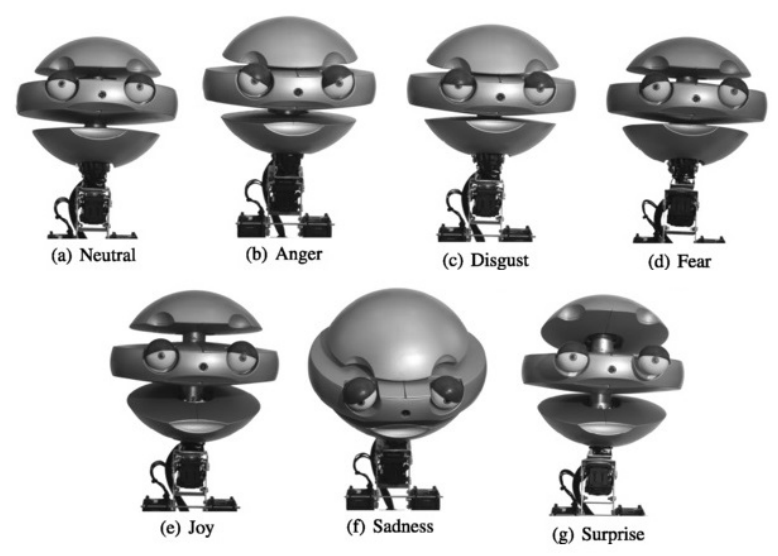

Figure 2. Facial expressions by EMYS robot.

\section{Post-Interaction}

After they ended the game, participants were asked to fill a questionnaire regarding their interaction with the robots. First, participants were asked to evaluate their emotional state after the interaction. Second, participants were asked to make a self-evaluation of the assertiveness level that they displayed during the interaction. Participants were also asked to assess their perception of the robots and the robots' level of assertiveness. Each of the set of questions referring to the robots was answered twice (once regarding each robot). We gave different names to each robot so that participants could distinguish

\footnotetext{
${ }^{4}$ The utterances used in each condition varied in each decision point obeying to Table 1 configurations.
}

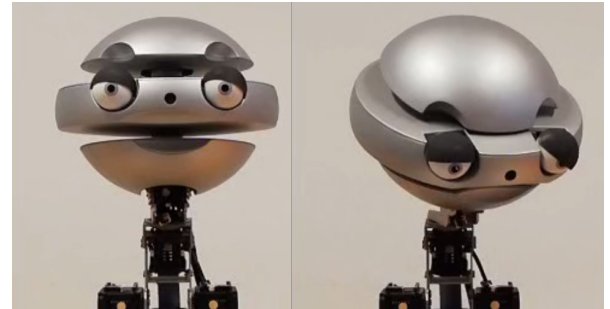

Figure 3. EMYS robot with postures pride at left and shame at right.

them. The entire experiment took approximately 60 minutes to complete, and all participants received a cinema ticket worth around $€ 6$ as a reward for their participation.

\section{Materials}

In order to analyse the effect of the level of assertiveness displayed by the NPCs, we conducted a quantitative study using two autonomous NPCs. For this purpose, we used two EMYS heads ${ }^{5}$ with a similar embodiment, programmed to display different levels of assertiveness and act as advisers in an interactive storytelling scenario. We chose two EMYS robots to autonomously interact with participants due to the peculiar design of the robot, in particular, its capability to display facial expressions simulating emotional feelings as seen in Fig. 2.

A touchscreen was used to display the interactive story and to enable the user to interact and chose her/his path in the story. Besides that, a digital female voice generated by a textto-speech application was used to narrate the scenes of the story. Moreover, next to each robot, a speaker was placed in order to transmit the robot's verbal utterances (male voice), ensuring that the sound would come from the direction of the robot talking.

\section{Manipulation}

The manipulation of the level of assertiveness displayed by each robot was achieved through the manipulation of four physical aspects of the robots' behaviour; namely (a) pitch, (b) rate of speech, (c) posture, and (d) eye gaze behaviour (see Table 1).

Table 1. Robots/NPCs configurations.

\begin{tabular}{c|c|c|c}
\hline & $\begin{array}{c}\text { Neutral } \\
\text { Robot }\end{array}$ & $\begin{array}{c}\text { Assertive } \\
\text { Emys }\end{array}$ & $\begin{array}{c}\text { Less-Assertive } \\
\text { Glin }\end{array}$ \\
\hline Pitch & default & X-low & X-high \\
\hline Rate & medium & $+20 \%$ & medium \\
\hline Posture & neutral & pride & shame \\
\hline Gaze & $50-50$ & $\begin{array}{c}\text { more to } \\
\text { the player }\end{array}$ & $\begin{array}{c}\text { less to } \\
\text { the player }\end{array}$ \\
\hline
\end{tabular}

Following the findings and validation presented in [49], the voice and posture of the robots were manipulated to convey different levels of assertiveness. The manipulated parameters in the robot's voice were pitch (with values x-low, default and

\footnotetext{
${ }^{5}$ For more information, see: https://emys.co/
} 
$\mathrm{x}$-high) and speech rate (values set as medium and $+20 \%)^{6}$. We also implemented three different postures for the robots: (a) neutral, (b) pride and (c) shame. The neutral posture exhibits the robot with head and eyebrows in a levelled position. Differently, in the pride posture (see Fig. 3, left) the robot presents the head in an elevated position, and the eyebrows are more open than in the neutral pose. In the shame posture (see Fig. 3 right), the robot's head is tilted down, and the eyebrows are also leaning down. In addition, the robots were programmed to display congruent eye gaze behaviours. In particular, in the test condition, in which the NPCs display assertiveness, the NPC with a higher level of this trait gazes more often to the player than the robot displaying low levels of this trait. In the control condition, where the NPCs have a neutral posture, the agents were programmed to direct their gaze towards the player half of the time; and the other half towards a random point in the room.

Furthermore, since both robots had the same embodiment, we also used the names suggested in [49] in order to ease their distinction by the participants. In this context, the robotic NPC displaying higher levels of assertiveness was called Emys, whereas the one displaying low levels of this trait was named Glin.

Finally, we manipulated the feedback given by the robot to the player after the latter has chosen an option. When the players' decision is not congruent with the suggestions given by the robot, the robot will display anger (moving its head back and forward and frowning its eyebrows). When the players' decision is congruent with the path suggested by the robot, the robot will display happiness (nodding yes).

\section{INTERACTIVE STORYTELLING PLATFORM}

The platform of our game was developed using the language C\#, which allows the integration with the framework [57] that supports the communication with our physicals NPCs. The interactive storytelling platform is composed by the scene generator, the persuasion module, the robot selection function and the personality module. Fig. 4 illustrates how the different modules are connected and how the player interacts with the system.

\section{Scene Generator}

The Scene Generator is an essential part of this platform, and determines what is going to be the next scene of the story flow (see Fig. 5) according to the player's final decision.

Our story follows the parallel interactive storytelling structure with two endings. That structure has the characteristic of having different paths heading to a specific central DP with the same end to all paths. In our story, we have three of those fundamental points. However, before reaching those points, the player can go to different parts of the story and face different decisions depending on the choices made. In total, the story has 30 distinct DPs, and to reach the end, the player passes through a minimum of 20 and a maximum of 26 DPs.

\footnotetext{
${ }^{6}$ Pitch values ( $\mathrm{x}$-low, low, medium, high, $\mathrm{x}$-high, or default) and rate values (x-slow, slow, medium, fast, $x$-fast, or default) were chosen from the prosody elements present in https://www.w3.org/TR/speechsynthesis/\#S3.2.4
}

The Scene Generator is responsible for showing the selected scene for each DP and call the text-to-speech to process the corresponding utterances for the narrator. The narrator finishes presenting the scene, then the user is faced with the two possible options and must inform of his/her intention.

Fig. 5 depicts a small part of the scheme that represents the full story with the DPs and the MBTI dimension that is measured by it (more details in subsection 5.4). For example, when the story begins, the first DP (DP1) measures the dimension Extroversion/Introversion (E/I) and depending on the player's final decision on this point the story can follow the path in DP2 that measures the dimension Thinking/Feeling (T/F) or the path in DP3 that measures the E/I dimension again.

\section{Persuasion Module}

The Persuasion Module receives the user's intention and according to the System Settings, generates the persuasive gestures. This strategy was employed since we want to have a way to measure if the persuasion techniques designed were successful or not after the user final decision.

This model uses information related to the user and the robots personality inserted in the system ahead of the user interaction. The user personality was collected with the MBTI questionnaire in the pre-interaction phase, and the robot personality was selected according to the condition to be tested (assertiveness high and low or neutral characteristics). With the player and the NPCs' characteristics, this module determines which type of persuasion (verbal and non-verbal cues) the NPCs should do. The Non-Verbal Cues prepared are the facial expressions (associated with the emotions) and head movement (as nodding as a response to a decision made congruently to what the NPC "wishes"). The Verbal Cues are utterances mentioned by the NPCs with hints after the intention is pointed out by the player, trying to influence s/he to change or maintain the decision. Additionally, this module can manage the intensity with which the robot can perform these cues. For instance, the facial expression that represents anger has levels

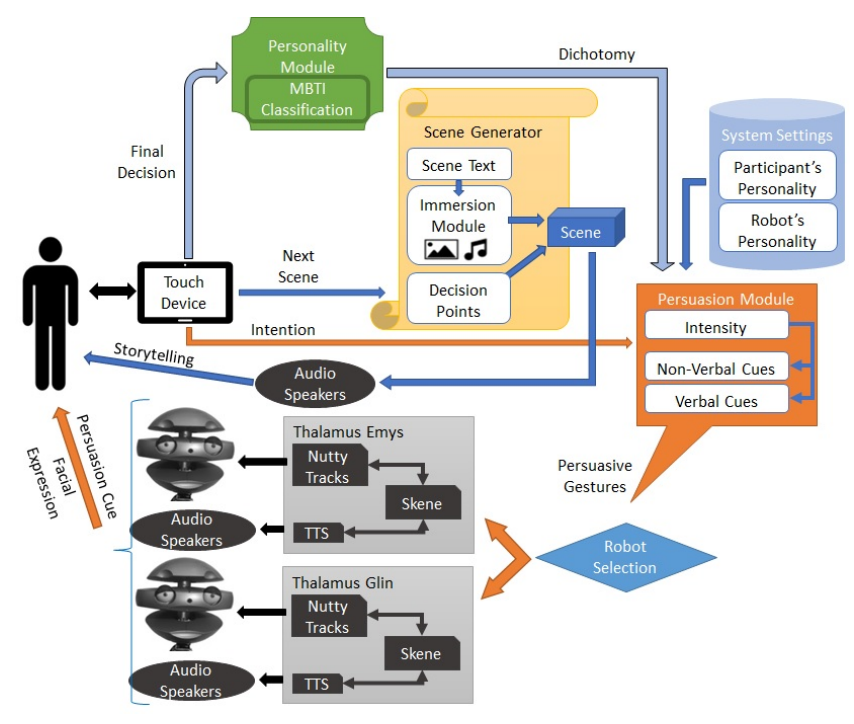

Figure 4. Storytelling Platform. 


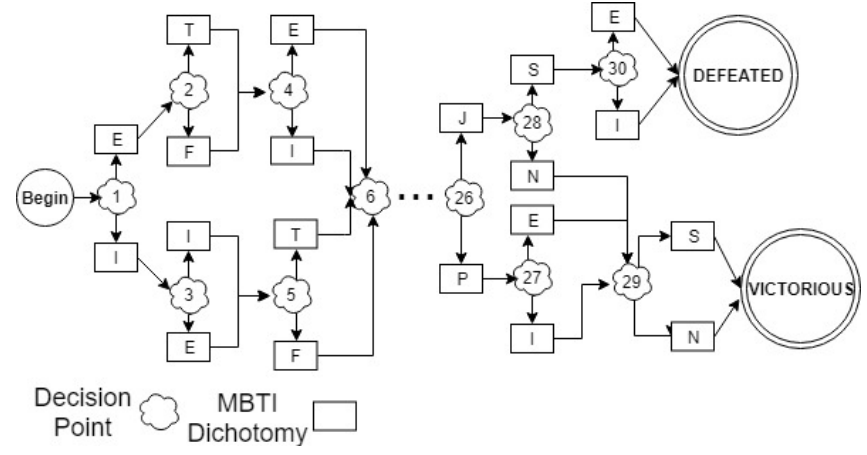

Figure 5. Scheme of the story flow.

of intensity, where the robot's joints can open more or less, as well as changes in velocity and angle of the robot movements. The intensity of the cues is defined by the number of times the player was congruent or incongruent with the NPC's "wish".

The definitions of the gestures are sent to the NPC by the Robot Selection function (subsection 5.3) that determines which NPC is going to perform the first persuasive gestures. So, depending on the NPC, the respective function is called according to the framework created by Ribeiro et al. [57]. That framework is part of an ecosystem composed of a model and tools for the integration of an AI agent with a robotic embodiment in HRI or a virtual character in HAI scenarios.

After the player final decision, this module is reactivated by the Personality module (subsection 5.4) that sends information about the personality classification and the final choice. With that activation, the persuasive gesture that the advising NPC will perform is generated. If the player's final choice was congruent with the player's personality, the NPC would perform an animation expressing its contentment with a joyful animation. Otherwise, the NPC would perform a sadness animation.

\section{Robot Selection Function}

During the story, each user will interact with the system and one of the two NPCs in each DP through a specific order. This order is defined by the Robot Selection function and uses what is defined in $\mathscr{G}$ in order to perform a decision.

$$
\mathscr{G}=\{\mathscr{P}, \mathscr{R}, \mathscr{C}\}
$$

Each element of $\mathscr{G}$ will correspond to the personality trait being presented $\mathscr{P}$, the personality simulated by the NPC selected $\mathscr{R}$ and the NPC congruence with the player's personality $\mathscr{C}$.

$\mathscr{P}$ is defined with the four dichotomies of the MBTI questionnaire, Extroverted-Introverted (EI), Sensing-iNtuition (SN), Thinking-Feeling (TF) and Judging-Perceiving (JP).

$$
\mathscr{P}=\{E I, S N, T F, J P\}
$$

$\mathscr{R}$ is defined with the personalities that each NPC would use, Assertive (A) and Less-Assertive (LA).

$$
\mathscr{R}=\{A, L A\}
$$

$\mathscr{C}$ is defined with the information if the NPC will act in favour of the player's personality (F) or opposite (against) the player's personality $(\mathrm{O})$.

$$
\mathscr{C}=\{F, O\}
$$

Each element $o$ of the personality traits $\mathscr{P}$ will have a maximum number of decision points associated with each dichotomy pair of the MBTI, designated as $k$. This way, each element $j$ of the NPCs personality $\mathscr{R}$, will correspond to one element $p$ of the NPC's congruence $\mathscr{C}$. This combination will be repeated for $k$ times to each dichotomy pair present in $\mathscr{P}$.

$$
\mathscr{P}_{o}=\left\{R_{1} C_{1} ; R_{1} C_{2} ; R_{2} C_{1} ; R_{2} C_{2} ; \ldots ; R_{j} C_{p}\right\}
$$

Let's consider $\mathscr{G}$ as the following:

$$
\mathscr{G}=\left\{\left\{P_{E I} ; P_{S N} ; P_{T F} ; P_{J P}\right\}\left\{R_{A} ; R_{L A}\right\}\left\{C_{F} ; C_{O}\right\}\right\}
$$

For instance, analysing the dichotomy pair $E I$ and having five decision points in the story $(k=5)$ to measure this pair, we would have $P_{E I}$ as being

$$
\mathscr{P}_{E I}=\left\{A_{1} F_{1} ; A_{1} O_{2} ; L A_{2} F_{1} ; L A_{2} O_{2} ; A_{1} F_{1}\right\}
$$

As such, when the player is at a DP that is measuring the $E I$ dichotomy, the selected NPC's personality is going to be the first of the list from this dichotomy in which the personality is opposite to the last one selected for the previous pair (not necessarily EI preference). For example, if the last personality selected was assertive for the pair JP, the next NPC's personality needs to be a less-assertive one. This way we can balance the number of times each NPC, presenting different levels of assertiveness, interacts with the participant during the story.

Then, the selected NPC performs the persuasion attempt and, depending on whether the player's intention is congruent or not with the players' personality, the NPC will try to convince $\mathrm{him} /$ her to change or maintain their decision. For example, if the player is classified as being an extrovert, but selects a decision that is more congruent with an introverted personality profile, the NPC will try to convince the player to change his/her decision. After this first persuasion attempt, the player must decide if he/she is going to change his/her decision based on the NPC suggestion or disregard the persuasion attempt and stick to their original intention. Then, the player's personality classification and the dichotomy regarding the final decision made (in this example 'I' stands for Introverted or ' $E$ ' stands for Extroverted) are sent to the Personality Module (PM).

\section{Personality Module}

The Personality Module (PM) receives the dichotomy associated with the DP and measures the player's personality classification for each decision in real-time. This classification was developed through a parallel mechanism based on the findings in [48]. In [48], the authors explained how they conceived an interactive story having decision points that are "connected" to each dichotomy pair of the MBTI questionnaire. Their classification was made having into consideration the fact that the MBTI presents hypothetical situations to the respondents and then gives different response options. Each response option presents a behaviour in which different levels of one of the four personality dimensions are more predominant than the others. The score of all responses is then attributed according to the preference of respondents to typically choose behaviours that 
are predominantly representative of one of the dimensions (e.g. Extroverted/Introverted). In this sense, we devised a story that follows the same principles of [48] as seen in Fig. 5, that considers all MBTI dimensions, and balances the measurement of these dimensions by dividing the total number of DPs equally among dimensions of the MBTI.

In this way, it is possible to simulate those situations presented in the MBTI questionnaire in a game scenario. For example, the personality questionnaire has a question that asks if a person would prefer to speak in public or in private. In this sense, it is possible to simulate the situation of speaking in public showing a DP to the player with the same topic, for instance, the DP1 aforementioned (if s/he would like to speak to the people or gather the council).

For each DP, after the player final decision, the PM will activate the Persuasion Module again by sending the player's personality classification for the DP and the final decision selected. Finally, the process starts over again, with the Scene Generator calling the text-to-speech to process the utterances of the next scene, creating the scene with all components and showing the respective options.

With the features described before, at the end of the game, the system presents information about both (a) the game outcome (victory or defeat) and (b) the MBTI dimensions score of each player based on the decisions made in each DP.

\section{DESCRIPTIVE ANALYSIS OF THE RESULTS}

Overall, we analysed a total of 1220 decision points. As previously stated, at each DP, participants were given the opportunity to change their decision once after hearing the NPC's advice. In $84,4 \%$ (i.e. 1078) of the DPs, participants did not alter their response after the NPC's persuasion attempt. The majority of the participants that decided not to change their decision after the NPCs' intervention (approx. 75\%), rated themselves high in assertiveness, whereas most of the participants that opted for making a change in their final choice (approx. 70\%) rated themselves low in this trait. In total, approximately $72 \%$ of our participants rated themselves high in assertiveness, whereas the remaining $28 \%$ reported low levels of assertiveness (see Table 2).

Table 2. Participants' assertiveness level and decision change.

\begin{tabular}{c|c|c}
\hline $\begin{array}{c}\text { Participant } \\
\text { Assertive Level }\end{array}$ & $\begin{array}{c}\text { Changed } \\
\text { Decision }\end{array}$ & $\begin{array}{c}\text { Not Changed } \\
\text { Decision }\end{array}$ \\
\hline High (72\%) & $25 \%$ & $75 \%$ \\
\hline Low (28\%) & $70 \%$ & $30 \%$ \\
\hline
\end{tabular}

In addition, given that one of the NPCs intervened at each DP, a total of 1277 interventions were made by those. Of this total, half was uttered by the NPCs displaying an assertive $(25 \%)$ and a less-assertive $(25 \%)$ personality trait in the test condition. The remaining interventions were uttered by the neutral NPCs in the control condition.

Finally, the scales for positive emotions and the scale for assertiveness used in the pre-questionnaire presented good to acceptable levels of reliability (the database and Cronbach alphas can be consulted in [50]. The scale components used to measure negative emotions and the level of assertiveness post-interaction presented suboptimal levels of consistency.

\section{Manipulation Verification}

In order to verify that both NPCs were perceived differently regarding their levels of assertiveness, we conducted a manipulation verification. In particular, we conducted a paired samples t-test, which yielded significant differences in the perception of assertiveness between the NPCs $(t(60)=2.55, p=.01)$. More specifically, Emys was perceived as being more assertive $(M=5.20 ; S D=.65)$ in comparison to Glin $(M=2.54 ; S D=$ $.74 ;$ Cohens $\left.^{\prime} d=0.99\right)$.

\section{Hypothesis Test}

\section{Participant's Emotional State - $\mathrm{H} 1$}

In order to analyse this hypothesis, we computed two variables corresponding to the average of all positive (interested, enthusiastic, inspired, active and determined) and negative emotions (nervous, fearful, scared, guilty and frightened). To analyse whether there was a difference between the feelings reported by participants after interacting with the assertive versus less-assertive NPC, we conducted two paired samples t-test for each category of emotions (positive and negative). The results for the positive dimension yielded a statistically significant difference between the two conditions $(t(29)=-3.78 ; p<.001)$. More specifically, Glin (lessassertive NPC) was associated with a higher level of positive emotions $(M=4.2 ; S D=.09)$ than the assertive NPC, Emys $\left(M=3.7 ; S D=.13 ;\right.$ Cohens $\left.^{\prime} d=.84\right)$. However, no statistical difference was found when comparing the negative emotions reported by participants after interacting with either one of the NPCs $(t(29)=.67 ; p=51)$. In general, participants reported very low levels of negative emotions when interacting either with Emys $(M=1.4 ; S D=.07)$ or Glin $\left(M=1.3 ; S D=.07 ;\right.$ Cohens $\left.^{\prime} d=.27\right)$.

Players' Choice to Change Decision and Favourite NPC - H2 To analyse the relation between the NPC's display of personality (versus neutral) and the participants' choice to change their decisions in the game, we conducted a McNemar test with repeated measures. We found a statistically significant difference in proportion of decision changes between the two conditions $(\operatorname{McNemar}(2)=256.81, p<.001)$. In particular, when interacting with the robots displaying personality, we found that $83.0 \%$ of the participants kept their initial decisions, whereas $17.0 \%$ of them changed their final decision. For the NPCs without personality (control condition), the participants kept their decisions in $87.8 \%$ and changed their final decision in $12.2 \%$.

\section{Assertiveness and Decision-making - H3}

To test this hypothesis, we first created two dichotomous variables, the first reflecting the player's choice to change their decision or not and the second, related to the level of the participants' self-reported assertiveness. To create the latter variable we categorised all participants that scored beneath the middle point of the scale as having a low level of assertiveness and all the remaining participants as having a high level of assertiveness. We then conducted a KolmogorovSmirnov test to investigate the assumption of normality and 
observed acceptable levels of fitness to the normal distribution $(K-S(61)=.16 ; p>.05)$. Then, we conducted a binary regression model using the Block entry method in which we tested the effectiveness of both the level of assertiveness of the participant and the level of assertiveness displayed by the NPC in predicting players' choice to change their decision in the game. Our null model was not significant $(\operatorname{Wald}(1)=2.59 ; p=.11)$. Additionally, our analysis showed that neither the level of assertiveness of the participant $(\operatorname{Wald}(1)=.79 ; p=.38)$ nor the level of assertiveness displayed by the NPC $(\operatorname{Wald}(1)=2.34 ; p=.13)$ were good predictors of participants' choice to change their decision.

Exploratory Analysis: Game Experience and Game Behaviour We analysed if the frequency with which a participant plays games had any impact on the way people play. In particular, we conducted a $\chi^{2}$ in which we analysed the relationship between the frequency with which participants reported playing games (I do not usually play; I play sometimes and I reserve some of my time to playing games) and their intention to repeat the same game strategy that they used in the storytelling scenario if they were given the opportunity to play again. However, our analysis did not reveal any significant differences $\left(\chi^{2}(2)=\right.$ $4.48 ; p=.11)$. Among the participants who won the game, $24 \%$ reported that they would probably go for the same path if they could play again, and 15\% would opt for a different path. Among those who lost, $17 \%$ would likely follow the same path, and $44 \%$ would choose another path.

Moreover, we also analysed the difference between the everyday level of assertiveness reported by participants and their evaluation of their level of assertiveness on our game scenario. To do this, first, we assessed the normality of our variables with a Kolmogorov-Smirnov test $(K-S(61)=.09 ; p=.200)$ for the assertiveness in the pre-test and $(K-S(61)=.13 ; p=$ .012 ) for the post-test. Finally, we performed a paired sample t-test comparing the assertiveness levels reported by the participants. The t-test results show statistically significant differences between the assertiveness reported in the pre and post-questionnaires $(t(60)=-4.55 ; p<.00)$. Plus, the assertiveness reported by the participants before the interaction with the NPCs $(M=3.39 ; S D=.48)$ was lower than the level of assertiveness that they reported during the game $(M=3.83 ; S D=.34)$.

In addition, we also tested the relationship between the level of assertiveness in everyday scenarios of the participant (high or low) and their preference towards a specific robot and found that, in our sample, these variables seem to present an independent distribution $\left(\chi^{2}(3)=4.48 ; p=.214\right)$. As an exploratory analysis, we also analysed participants preferences towards each of the NPC according to the outcome of the game (win or lose) and found no differences in participants' preference towards the assertive or the less assertive $\operatorname{robot}\left(\chi^{2}(2)=1.16\right.$; $p=.56)$.

\section{DISCUSSION}

Our study yielded interesting findings regarding how the display of personality traits by robotic NPCs can influence the player's emotional state and the participants' levels of assertiveness during a game that demands decision-making actions. Our results from the manipulation verification revealed that the players that interacted with NPCs with different assertiveness levels could understand that they were in the presence of NPCs with distinct traits. This finding validates our trait manipulation, reinforcing the fact that the configuration of a high level of assertiveness and low level of assertiveness suggested in [49] contributed to this clear distinction between the NPCs. This finding can be useful, for instance, in scenarios in which the game developer wants to persuade a player to accept a specific mission or to follow a determined path.

Plus, our findings also suggest that participants report more positive emotional responses towards a NPC that displays low levels of assertiveness. This is contrary to our $\mathrm{H} 1$, which predicted participants' would respond more positively towards robotic NPCs displaying high levels of assertiveness. Although future investigation is necessary to fully explain this finding, one possible explanation is that participants perceived the high assertiveness robot as being more overbearing or pushy than the low assertiveness robot. However, we found no difference in terms of negative emotional responses. The lack of an effect here, symmetric to the one found for the positive emotions, might have been due to a floor effect, given that the negative feelings towards the robots reported by participants were very low (below the middle point of the scale).

Furthermore, we also observed that participants who interacted with robots displaying different levels of assertiveness (high or low) changed their decisions in the game more often than those who interacted with robots displaying a neutral level of this trait. These results provide evidence in favour of our $\mathrm{H} 3$ and are congruent with past literature demonstrating that virtual and embodied characters can be perceived and responded to in similar ways to their human counterparts and can thus, be effective persuaders [59, 45]. However, we did not observe an effect of the level of assertiveness displayed by the robot. Indeed, despite the fact that the persuasion strategies adopted in this study took into account the player's personality, the number of effective persuasion attempts were low in comparison with the total number of attempts.

In the majority of cases where the robots' persuasion attempt was successful (as measured by the participant changing his/her decision), the persuasion attempt conducted by the robot went in a direction that was parallel to the personality of the users, thus serving as a reinforcement to their behavioural tendencies. Reviews of the previous research on persuasion have consistently modelled persuasion (i.e. the degree of oscillation of personal beliefs) as a function of a set of classic variables that included the nature of choice (dichotomous or complex) and the discrepancy between the choice made by the individual and his prior beliefs $[17,16]$. These two variables might contribute to explain the difficulty to induce change or persuade an individual to act in ways that are not congruent with his personality observed in this study. In particular, it is thought that a discrepancy between an individuals' choice and the individuals' personality might hinder the persuasion process by causing a certain level of cognitive dissonance [17, 
16]. In fact, many approaches to increase persuasion leading to effective behaviour have resulted in the development of techniques aimed at reducing proximal and distal dissonance from the decision-making process, in an attempt to increase the effectiveness of the persuasion attempts [17, 27]. In our study, this effect might have been exacerbated by the important nature of the decisions the individual was asked to make (as these decisions could either lead to the success or defeat of his country) and the role that was attributed to him (leader of that country). Furthermore, the fact that the individual was primed to take a position of power (leader) might have also hindered the persuasion attempts conducted by the robot (c.f. [60]).

In addition, some authors have also suggested that the type of decision that the individual is required to make might, by itself, affect the effectiveness of persuasive communication attempts (e.g. [17, 46]. This is particularly true for choices that are presented in a dichotomous manner and that require the individual to locate their decision on an important matter on one of two opposing sides. Models of response or decision that express a nearly continuous array of choices facilitate persuasion by allowing the individual to compromise between two opposing or incongruent points of view [17]. In our scenario, individuals were requested to make binary decisions at multiple points of the interactive story, which might have hinder the effectiveness of the persuasion attempts made by the robots.

Furthermore, our results can also be partially explained by the fact the most of the participants in our study considered themselves to have a high level of assertiveness. In particular, this fact might lessen the likelihood of participants to change their decisions due to previous research that suggests that people who consider themselves more assertive tend to be more confident about their decisions [31].

Regarding the exploratory analysis we conducted, we also observed that the previous level of gaming experience possessed by the participants did not affect their attitudes regarding the re-evaluation of their game strategy. Indeed, even considering the final outcome of the game (winning or loosing), we still did not observe a difference in this regard. This might have been partially due to the interactive plotline created for this study, which might have boosted the curiosity and sense of adventure of players. Moreover, this result can also be explained by the lack of a reward associated with the game outcome. All players were explained, at the start of the experiment, that they would receive a compensation for participating in the study, regardless of their actual performance. Several studies have suggested the existence of an effort-reward balance and argued that this balance can have a regulatory effect in the motivation and performance of participants in research studies $[41,58]$. In this sense, because participants in our study did not receive benefits associated with improved performance (winning), they might have disregarded the game outcome in favour of other strategy-related criteria to re-evaluate their game behaviour.

Furthermore, our results also suggested that there were differences between the self-reported level of assertiveness of participants in everyday life and the levels of assertiveness that they reported in our game. More specifically, participants reported higher levels of assertiveness when playing our game than when they were in other everyday scenarios. These results might be partially explained by a priming effect of the instructions and the role assigned to the participant (i.e. the leader of a country) (c.f. [60]).

Overall, this study provides evidence in favour of the importance of the psychological humanization of robots in gaming contexts, through the display of personality traits. However, it leaves open the question of which task-specific traits present a larger potential to enhance HRI and improve the persuasion abilities displayed by robots. In addition, we also explored the potential effect of the employment of personalised persuasion tactics and, despite not having found any effects in this regard, contribute to the literature by presenting an interactive game scenario that considers the user personality, which can be used in future research.

\section{FUTURE WORK AND IMPLICATIONS FOR PLAYER EXPE- RIENCE AND GAME DESIGN}

Games are a useful tool to study a wide range of human behaviours and its use in this context has increased in the last few decades [23]. Moreover, games are also a persuasive form of entertainment for millions of people around the world and have been demonstrated to have potential in areas such as education [41], promotion of healthy behaviours [53, 25] and sustainable habits [36]. Many of these uses for games require the user to make decisions during the game, and many involve the player interacting with others (whether it is other human players, virtual NPCs or in this case socially embodied robots). In this context, we developed a game that attempts to leverage the use of personalized interaction techniques based on the players' personality (in particular, their level of assertiveness) and we used this game to understand how personalization could improve the robots' persuasive abilities in the context of the game, as well as the overall game experience.

Our findings revealed that robotic NPCs that displayed personality traits were able to influence the players' emotional state and their level of assertiveness in the game. These findings can be of use in other areas involving interaction with robots. For instance, previous literature has underlined the potential of robots in improving user' motivation in areas like care, education and pro-social behaviours [12]. Robots displaying personality features can be used to augment motivation to engage in this type of behaviours. From a player experience standpoint, the adaptation of the personality traits displayed by the robot to those exhibited by the user can also foster the development of better relationships between humans and robots $[63,18]$. This is supported by multiple psychological models that are relevant in the domain of player experience and user satisfaction (c.f. [63]) and thus, is of central importance in the context of game development and evaluation.

In terms of future research, the authors would like to call for future work evaluating how previous findings of persuasion in HRI translate to real-life environments and what are the main ethical implications that should be taken in consideration when developing robots aimed at influencing the users' behaviour. In 
the specific context of games involving robots, further research on the role of the display of other personality traits (such as extroversion or openness) in increasing the effectiveness of persuasion attempts aimed at altering the behaviour or attitudes of users and the quality of their gaming experience is also necessary.

\section{ACKNOWLEDGMENTS}

We would like to thank the National Council for Scientific and Technological Development (CNPq) program Science without Border: 201833/2014-0 - Brazil and Agência Regional para o Desenvolvimento e Tecnologia (ARDITI) - M1420-09-5369000001, for $\mathrm{PhD}$ grants to first and second authors respectively. This work was also supported by Fundação para a Ciência e a Tecnologia: (FCT) - UID/CEC/50021/2013 and the project AMIGOS:PTDC/EEISII/7174/2014.

\section{REFERENCES}

[1] R. Alberti and M. Emmons. 2017. Your Perfect Right: Assertiveness and Equality in Your Life and Relationships. New Harbinger Publications.

[2] M. Astrid, N. Krämer, and J. Gratch. 2010. How our personality shapes our interactions with virtual characters-implications for research and development. In International Conference on Intelligent Virtual Agents. Springer, 208-221.

[3] S. Bakkes, C.T. Tan, and Y. Pisan. 2012. Personalised gaming: a motivation and overview of literature. In Proceedings of the 8th Australasian Conference on Interactive Entertainment: Playing the System. ACM, 4.

[4] I. Baroni, M. Nalin, M. Zelati, E. Oleari, and A. Sanna. 2014. Designing motivational robot: How robots might motivate children to eat fruits and vegetables. In Robot and Human Interactive Communication, 2014 RO-MAN: The 23rd IEEE International Symposium on. IEEE, 796-801.

[5] C. Bartneck, D. Kulić, E. Croft, and S. Zoghbi. 2009. Measurement instruments for the anthropomorphism, animacy, likeability, perceived intelligence, and perceived safety of robots. International journal of social robotics 1, 1 (2009), 71-81.

[6] S. Berkovsky, J. Freyne, and H. Oinas-Kukkonen. 2012. Influencing individually: fusing personalization and persuasion. ACM Transactions on Interactive Intelligent Systems (TiiS) 2, 2 (2012), 9.

[7] E.P. Bernier and B. Scassellati. 2010. The similarity-attraction effect in human-robot interaction. In 2010 IEEE 9th International Conference on Development and Learning. IEEE, 286-290.

[8] M. Busch, J. Schrammel, and M. Tscheligi. 2013. Personalized persuasive technology-development and validation of scales for measuring persuadability. In International Conference on Persuasive Technology. Springer, 33-38.

[9] D. Byrne, G.L. Clore, and G. Smeaton. 1986. The attraction hypothesis: Do similar attitudes affect anything? (1986).
[10] Z. Callejas, D. Griol, and R. López-Cózar. 2014. A framework for the assessment of synthetic personalities according to user perception. International Journal of Human-Computer Studies 72, 7 (2014), 567-583.

[11] A. Cerekovic, O. Aran, and D. Gatica-Perez. 2014. How do you like your virtual agent?: Human-agent interaction experience through nonverbal features and personality traits. In International Workshop on Human Behavior Understanding. Springer, 1-15.

[12] V. Chidambaram, Y. Chiang, and B. Mutlu. 2012. Designing persuasive robots: how robots might persuade people using vocal and nonverbal cues. In Proceedings of the seventh annual ACM/IEEE international conference on Human-Robot Interaction. ACM, 293-300.

[13] A. Chowanda, P. Blanchfield, M. Flintham, and M. Valstar. 2016. Computational Models of Emotion, Personality, and Social Relationships for Interactions in Games: (Extended Abstract). In Proceedings of the 2016 International Conference on Autonomous Agents \& Multiagent Systems (AAMAS '16). International Foundation for Autonomous Agents and Multiagent Systems, Richland, SC, 1343-1344.

http://dl. acm.org/citation. cfm?id=2936924.2937151

[14] P. Costa and R. McCrae. 1992. Revised NEO personality inventory (NEO PI-R) and NEP five-factor inventory (NEO-FFI): professional manual. Psychological Assessment Resources Lutz, FL.

[15] J. Crawford and J. Henry. 2004. The Positive and Negative Affect Schedule (PANAS): Construct validity, measurement properties and normative data in a large non-clinical sample. British journal of clinical psychology 43, 3 (2004), 245-265.

[16] J.P. Dillard and M. Pfau. 2002. The persuasion handbook: Developments in theory and practice. Sage Publications.

[17] J.P. Dillard and L. Shen. 2013. The Sage handbook of persuasion. Sage.

[18] Malte Elson, Johannes Breuer, and Thorsten Quandt. 2014. Know thy player: An integrated model of player experience for digital games research. Handbook of digital games (2014), 362-387.

[19] X. Fang and F. Zhao. 2010. Personality and enjoyment of computer game play. Computers in Industry 61, 4 (2010), 342-349.

[20] B.J. Fogg. 2002. Persuasive technology: using computers to change what we think and do. Ubiquity 2002, December (2002), 5.

[21] B.J. Fogg and D. Eckles. 2007. The behavior chain for online participation: how successful web services structure persuasion. In International Conference on Persuasive Technology. Springer, 199-209. 
[22] L. Francis, K. Monahan, and C. Berger. 1999. A laughing matter? The uses of humor in medical interactions. Motivation and emotion 23, 2 (1999), $155-174$

[23] G. Freedman and M. Flanagan. 2017. From dictators to avatars: Furthering social and personality psychology through game methods. Social and personality psychology compass 11, 12 (2017), e12368.

[24] J. Goetz, S. Kiesler, and A. Powers. 2003. Matching robot appearance and behavior to tasks to improve human-robot cooperation. In Proceedings of the 12th IEEE international workshop on robot and human interactive communication. IEEE Press Piscataway, NJ, 55-60.

[25] A. Grimes, V. Kantroo, and R.E. Grinter. 2010. Let's play!: mobile health games for adults. In Proceedings of the 12th ACM international conference on Ubiquitous computing. ACM, 241-250.

[26] J. Gu, T. Kim, and Y. Kwon. 2015. Am I Have to Extrovert Personality? An Empirical Investigation of Robot's Personality on the Two Contexts. Indian Journal of Science and Technology 8, 26 (2015).

[27] E. Harmon-Jones. 2002. A cognitive dissonance theory perspective on persuasion. The persuasion handbook: Developments in theory and practice 101 (2002).

[28] J.B. Hirsh, S.K. Kang, and G.V. Bodenhausen. 2012. Personalized persuasion: Tailoring persuasive appeals to recipients' personality traits. Psychological science 23, 6 (2012), 578-581.

[29] O. John, E. Donahue, and R. Kentle. 1991. The big five inventory: versions 4a and 54. (1991).

[30] M.C. Kaptein. 2012. Personalized persuasion in ambient intelligence. (2012).

[31] C.E. Kimble and S.D. Seidel. 1991. Vocal signs of confidence. Journal of Nonverbal Behavior 15, 2 (1991), 99-105. DOI : http://dx.doi.org/10.1007/BF00998265

[32] E. Knol and P.W. De Vries. 2011. EnerCities-A serious game to stimulate sustainability and energy conservation: Preliminary results. eLearning Papers 25 (2011).

[33] P. Lankoski and S. Björk. 2007. Gameplay Design Patterns for Believable Non-Player Characters.. In DiGRA Conference.

[34] S. LeBaron, J. Reyher, and J. Stack. 1985. Paternalistic vs egalitarian physician styles: the treatment of patients in crisis. The Journal of family practice (1985).

[35] K. Lee, W. Peng, S. Jin, and C. Yan. 2006. Can robots manifest personality?: An empirical test of personality recognition, social responses, and social presence in human-robot interaction. Journal of communication 56, 4 (2006), 754-772.

[36] I. Leite, A. Pereira, C. Martinho, and A. Paiva. 2008. Are emotional robots more fun to play with?. In
RO-MAN 2008-The 17th IEEE International Symposium on Robot and Human Interactive Communication. IEEE, 77-82.

[37] S.B. Linek, D. Schwarz, G. Hirschberg, M. Kickmeier-Rust, and D. Albert. 2007. Designing the non-player character of an educational adventure-game: the role of personality, naturalism, and color. In Proceedings of the International Technology, Education and Development Conference.

[38] M. Lorr and W.W. More. 1980. Four dimensions of assertiveness. Multivariate Behavioral Research 15, 2 (1980), 127-138.

[39] B. Mac Namee and P. Cunningham. 2001. A proposal for an agent architecture for proactive persistent non player characters. Technical Report. Trinity College Dublin, Department of Computer Science.

[40] A. Mileounis, R. Cuijpers, and E. Barakova. 2015. Creating robots with personality: The effect of personality on social intelligence. In International Work-Conference on the Interplay Between Natural and Artificial Computation. Springer, 119-132.

[41] T.R. Mitchell. 1973. Motivation and participation: An integration. Academy of Management Journal 16, 4 (1973), 670-679.

[42] C. Nass and K. Lee. 2000. Does computer-generated speech manifest personality? An experimental test of similarity-attraction. In Proceedings of the SIGCHI conference on Human Factors in Computing Systems. ACM, 329-336.

[43] C. Nass and Y. Moon. 2000. Machines and mindlessness: Social responses to computers. Journal of social issues 56, 1 (2000), 81-103.

[44] G.J. Ockey. 2009. The effects of group members' personalities on a test taker's L2 group oral discussion test scores. Language Testing 26, 2 (2009), 161-186.

[45] K. Ogawa, C. Bartneck, D. Sakamoto, T. Kanda, T. Ono, and H. Ishiguro. 2018. Can an android persuade you? Geminoid Studies: Science and Technologies for Humanlike Teleoperated Androids (2018), 235-247.

[46] Daniel J O'keefe. 2002. Persuasion. The International Encyclopedia of Communication (2002).

[47] A. Pacheco and C. Martinho. 2019. Alignment of Player and Non-Player Character Assertiveness Levels. In Proceedings of the 15th AAAI Conference on Artificial Intelligence and Interactive Digital Entertainment (AIIDE'19). ACM, to appear.

[48] R. Paradeda, M.J. Ferreira, C. Martinho, and A. Paiva. 2017. Using Interactive Storytelling to Identify Personality Traits. In Interactive Storytelling. ICIDS 2017. Lecture Notes in Computer Science, N. Nunes, I. Oakley, and V. Nisi (Eds.). Vol. 10690. Springer, Cham, 181-192. DOI :

http://dx.doi.org/10.1007/978-3-319-71027-3_15 
[49] R. Paradeda, M.J. Ferreira, C. Martinho, and A. Paiva. 2018. Communicating Assertiveness in Robotic Storytellers. In Interactive Storytelling. ICIDS 2018. Lecture Notes in Computer Science, vol 11318, R. Rouse, H. Koenitz, and M. Haahr (Eds.). Springer, Cham, 442-452. DOI :

http://dx.doi.org/10.1007/978-3-030-04028-4_51

[50] R.B. Paradeda, M.J. Ferreira, and R.A. Oliveira. 2019. Assertiveness in Storytelling Games. (Jul 2019). osf.io/hym56

[51] R.M. Perloff. 2017. The dynamics of persuasion: communication and attitudes in the 21 st century (6th ed.). Routledge Taylor \& Francis. https://content. taylorfrancis . com/books/download?dac $=C 2015-0-63789-2$

[52] R. Picard. 1995. Affective computing. Perceptual Computing Section, Media Laboratory, Massachusetts Institute of Technology (1995).

[53] J. Pollak, G. Gay, S. Byrne, E. Wagner, D. Retelny, and L. Humphreys. 2010. It's time to eat! Using mobile games to promote healthy eating. IEEE Pervasive Computing 9, 3 (2010), 21-27.

[54] A. Powers, S. Kiesler, S. Fussell, and C. Torrey. 2007. Comparing a computer agent with a humanoid robot. In Human-Robot Interaction (HRI), 2007 2nd ACM/IEEE International Conference on. IEEE, 145-152.

[55] C.W. Reynolds. 1999. Steering behaviors for autonomous characters. In Game developers conference, Vol. 1999. Citeseer, 763-782.

[56] T. Ribeiro, A. Pereira, A. Deshmukh, R. Aylett, and A. Paiva. 2014. I'm the mayor: a robot tutor in enercities-2. In Proceedings of the 2014 international conference on Autonomous agents and multi-agent systems. International Foundation for Autonomous Agents and Multiagent Systems, 1675-1676.
[57] T. Ribeiro, A. Pereira, E. Di Tullio, and A. Paiva. 2016. The SERA ecosystem: Socially Expressive Robotics Architecture for Autonomous Human-Robot Interaction. In Enabling Computing Research in Socially Intelligent Human-Robot Interaction: A Community Driven Modular Research Platform.

[58] B. Scott-Ladd, A. Travaglione, and V. Marshall. 2006. Causal inferences between participation in decision making, task attributes, work effort, rewards, job satisfaction and commitment. Leadership \& Organization Development Journal 27, 5 (2006), 399-414.

[59] M. Slater and M. Usoh. 1994. Body centred interaction in immersive virtual environments. Artificial life and virtual reality 1, 1994 (1994), 125-148.

[60] E.J. Strahan, S.J. Spencer, and M.P. Zanna. 2002. Subliminal priming and persuasion: Striking while the iron is hot. Journal of Experimental Social Psychology 38, 6 (2002), 556-568.

[61] A. Tapus and M. Matarić. 2008. User personality matching with a hands-off robot for post-stroke rehabilitation therapy. In Experimental robotics. Springer, 165-175.

[62] R.E. Van Heerden. 2017. Assertiveness and perception of style of assertiveness among future South African employees. Ph.D. Dissertation. University of the Free State.

[63] J. Wiemeyer, L. Nacke, Christiane Moser, and F. 'Floyd' Mueller. 2016. Player Experience. In Serious Games. Springer, Cham, Chapter 9, 243-271. DOI : http://dx.doi.org/10.1007/978-3-319-40612-1_9 